ARTICLE

https://doi.org/10.1038/s41467-021-23317-3

\title{
Evolution of spin excitations from bulk to monolayer FeSe
}

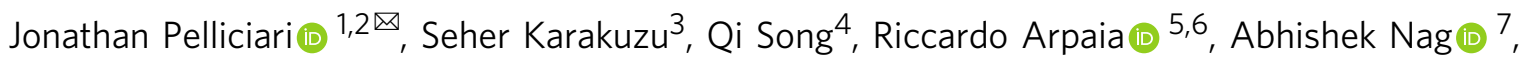
Matteo Rossi (1) ${ }^{5}$, Jiemin $\mathrm{Li}^{7}$, Tianlun $\mathrm{Yu}^{4}$, Xiaoyang Chen ${ }^{4}$, Rui Peng $\mathbb{B}^{4}{ }^{4}$, Mirian García-Fernández (10) ${ }^{7}$, Andrew C. Walters ${ }^{7}$, Qisi Wang (10 4, Jun Zhao (1) ${ }^{4}$, Giacomo Ghiringhelli (1) 5,8, Donglai Feng (1) 4, Thomas A. Maier (1) ${ }^{3,9}$, Ke-Jin Zhou (1) ${ }^{7}$, Steven Johnston ${ }^{10} \&$ Riccardo Comin ${ }^{1 凶}$

In ultrathin films of $\mathrm{FeSe}$ grown on $\mathrm{SrTiO}_{3}$ (FeSe/STO), the superconducting transition temperature $T_{\mathrm{C}}$ is increased by almost an order of magnitude, raising questions on the pairing mechanism. As in other superconductors, antiferromagnetic spin fluctuations have been proposed to mediate SC making it essential to study the evolution of the spin dynamics of FeSe from the bulk to the ultrathin limit. Here, we investigate the spin excitations in bulk and monolayer FeSe/STO using resonant inelastic x-ray scattering (RIXS) and quantum Monte Carlo (QMC) calculations. Despite the absence of long-range magnetic order, bulk FeSe displays dispersive magnetic excitations reminiscent of other Fe-pnictides. Conversely, the spin excitations in FeSe/STO are gapped, dispersionless, and significantly hardened relative to its bulk counterpart. By comparing our RIXS results with simulations of a bilayer Hubbard model, we connect the evolution of the spin excitations to the Fermiology of the two systems revealing a remarkable reconfiguration of spin excitations in FeSe/STO, essential to understand the role of spin fluctuations in the pairing mechanism.

\footnotetext{
${ }^{1}$ Department of Physics, Massachusetts Institute of Technology, Cambridge, MA, USA. ${ }^{2}$ NSLS-II, Brookhaven National Laboratory, Upton, NY, USA. ${ }^{3}$ Center for Nanophase Materials Sciences, Oak Ridge National Laboratory, Oak Ridge, Tennessee, USA. ${ }^{4}$ State Key laboratory of Surface Physics and Department of Physics, Fudan University, Shanghai, China. ${ }^{5}$ Dipartimento di Fisica, Politecnico di Milano, Milano, Italy. ${ }^{6}$ Quantum Device Physics Laboratory, Department of Microtechnology and Nanoscience, Chalmers University of Technology, Göteborg, Sweden. ${ }^{7}$ Diamond Light Source, Harwell Campus, Didcot, UK. ${ }^{8}$ CNRSPIN, Dipartimento di Fisica, Politecnico di Milano, Milano, Italy. ${ }^{9}$ Computational Sciences and Engineering Division, Oak Ridge National Laboratory, Oak

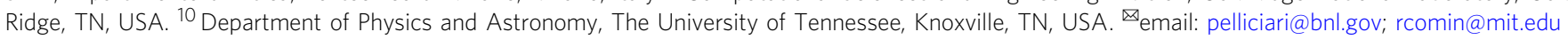


ron selenide (FeSe) occupies a unique place among Fe-based superconductors. It has the simplest structure, consisting of a square Fe lattice with Se ions situated above and below it, as depicted in Fig. 1a. It is superconducting with $T_{\mathrm{c}} \sim 8 \mathrm{~K}$ and has a structural transition at $T_{\mathrm{s}} \sim 90 \mathrm{~K}^{1-3}$. The Fermi surface of bulk FeSe is composed of cylindrical hole pockets at the $\Gamma$ point and elliptical electron pockets at the $M$ point (see Fig. 1c; hereafter, a Brillouin zone with two $\mathrm{Fe}$ sites per unit cell is adopted). The Fermi surface of FeSe/STO, on the other hand, is composed solely of circular electron pockets at the $M$ point $^{4-9}$, while the hole pockets at the $\Gamma$ point are pushed below the Fermi level (Fig. 1d). These observations are consistent with an electron doping of $\sim 0.1 / \mathrm{Fe}$, as extracted from the Luttinger count ${ }^{4,5,9}$, suggesting that STO acts as an electron donor for monolayer FeSe.

Simultaneous Néel- and stripe-like fluctuations have been observed in bulk FeSe at $\mathbf{q}=(1,0)$ and $(1,1)$ (reciprocal lattice units, r.l.u.), despite the lack of long-range antiferromagnetic order. These observations signal the presence of significant magnetic frustration that ultimately precludes any long-range order $^{3}$. From an experimental perspective, the investigation of spin excitations in FeSe/STO is complicated by the limited volume contributing to the magnetic scattering signal. Inelastic neutron scattering (INS) is currently unable to probe single atomic layers, and other light scattering techniques, such as Raman and optical spectroscopy, cannot disentangle the signals from the substrate, the FeSe layer, and the interface between the two. On this front, recent advances in Resonant Inelastic X-ray Scattering (RIXS) have allowed the detection of spin excitations in Fe-based superconductors, producing complementary information to INS ${ }^{10-18}$. The signal enhancement and sensitivity to electronic excitations that is afforded by resonant photoexcitation render RIXS a prime technique for investigating ultrathin materials. Additionally, the elemental selectivity of RIXS enables one to isolate the signal from specific atoms and disentangle the contributions from the film and the substrate. These aspects make
RIXS an ideal technique for studying magnetic excitations in FeSe/STO.

Here, we combine high-energy-resolution RIXS measurements and quantum Monte Carlo (QMC) calculations within the dynamical cluster approximation (DCA) to elucidate the spin dynamics of bulk FeSe and FeSe/STO films down to the single unit cell limit. We find that the magnetic excitations in FeSe/STO are gapped and dispersionless in momentum space, and harden significantly relative to other Fe-based superconductors. These observations are in stark contrast with the spin excitations of bulk FeSe, which exhibit an acoustic-like dispersion toward the zone center, similarly to other antiferromagnetic systems ${ }^{1}$. The evolution of the spin excitations is captured by DCA calculations of a bilayer Hubbard model ${ }^{19}$, which account for the transition from a two-band system into an incipient band system (see Methods). Correspondingly, we establish that the reconfiguration of the spin excitations from bulk to monolayer FeSe originates from the Lifshitz transition of the Fermi surface and accompanying loss of the hole pocket at the $\Gamma$ point. This transition quenches particlehole scattering processes, flattens and gaps out their dispersion, and increases their energy bandwidth, in agreement with the experimental observations.

\section{Results}

X-Ray absorption spectroscopy. Figure $2 \mathrm{a}, \mathrm{b}$ summarize the $\mathrm{Fe}$ $L$-edge X-ray Absorption Spectroscopy (XAS) data for bulk FeSe (FeSe hereafter) and monolayer FeSe (FeSe/STO hereafter), respectively. The XAS of FeSe resembles the spectra previously obtained from cleaved $\mathrm{Fe}$ pnictides crystals with $\mathrm{Fe}$ in a $2+$ oxidation state and embedded in a metal environment ${ }^{10,11,13,15,16}$. The XAS of FeSe/STO has an additional peak at higher energy, which could originate from new interfacial valence states induced by hybridization with orbitals of the STO substrate. The arrows in Fig. 2a, b specify the incident photon energies at which RIXS spectra were collected.
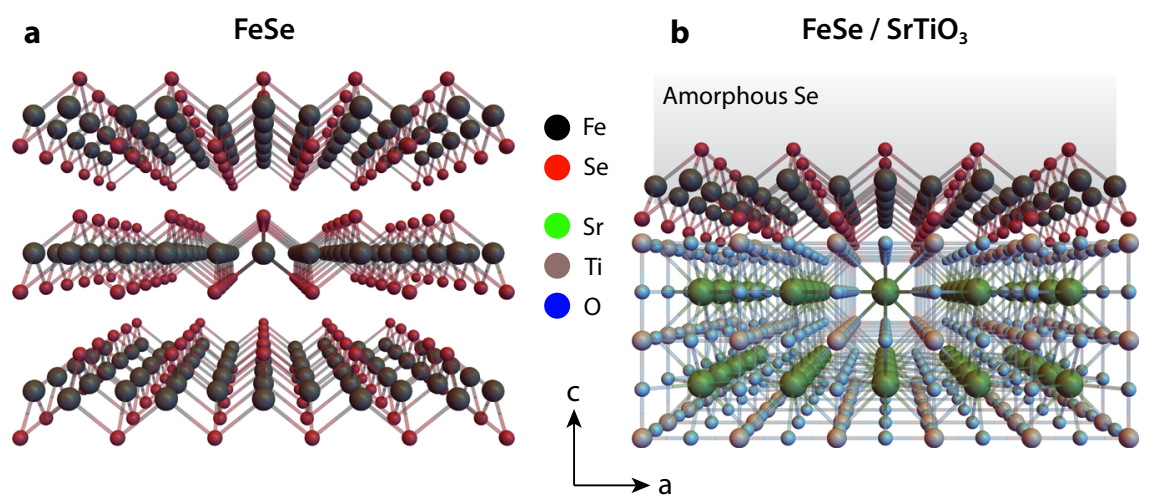

C

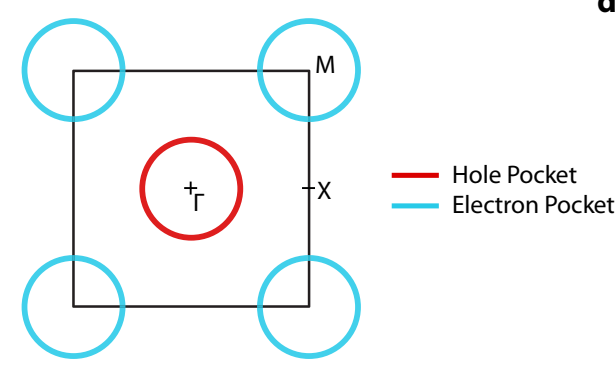

d

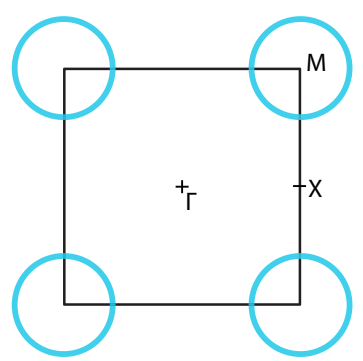

Fig. 1 Structure and Fermi surface of FeSe bulk and FeSe/SrTiO $\mathbf{3}$ (STO). a Structure of FeSe bulk. b Structure of FeSe/STO monolayer with Se capping. c, d Schematic Fermi surface of FeSe bulk (c) and FeSe/STO monolayer (d). The electron pocket of bulk FeSe has been drawn circular and not elliptical for simplicity and for correspondence with the theoretical model adopted here. 

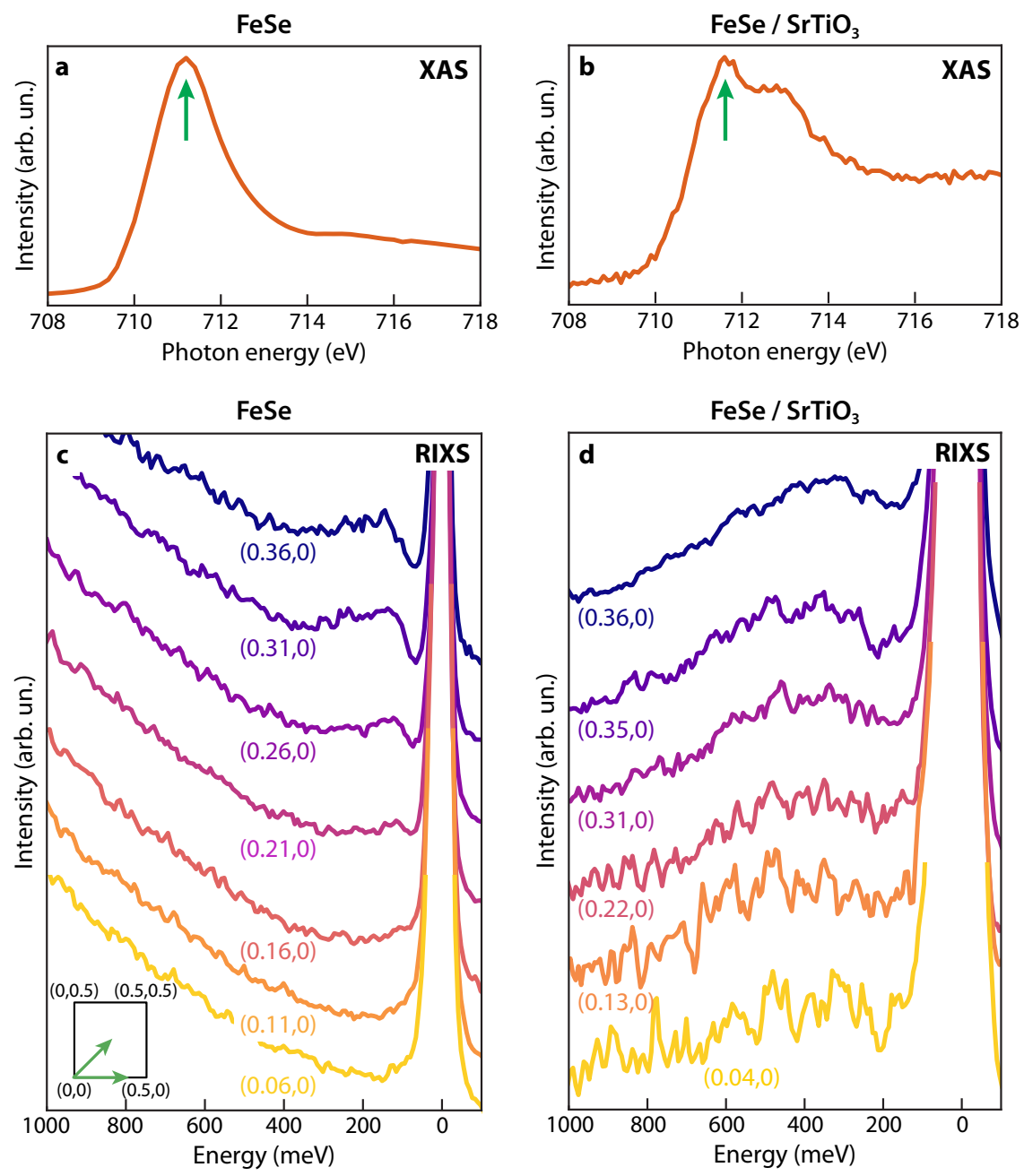

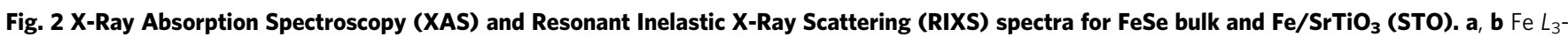
edge X-ray absorption spectra for FeSe bulk (a) and FeSe/STO (b), measured via total electron yield. The arrows mark the incident energy for the RIXS data displayed in $\mathbf{c}$ and $\mathbf{d}$. c, d High-energy resolution RIXS spectra of FeSe bulk (c) and FeSe/STO (d) at different momentum points along the highsymmetry direction $(0,0) \rightarrow(H, 0)$ [RIXS spectra along the $(0,0) \rightarrow(H, H)$ direction are reported in the Supplementary Information].

Resonant Inelastic X-Ray Scattering. Figure 2c, d show the corresponding high-resolution and high-statistics RIXS data on FeSe and monolayer FeSe/STO, respectively. In the bulk case, we detect a dispersive excitation at an energy of $\sim 140 \mathrm{meV}$ at $\mathbf{q}=$ $(0.36,0)$ r.l.u., which gradually decreases in energy toward the zone center until it merges into the elastic line. This mode is reminiscent of what observed in INS experiments ${ }^{3}$ and can be ascribed to spin excitations as previously shown in ref. ${ }^{14}$. A word of caution should be given, however, as FeSe lacks long-range antiferromagnetism, and, instead exhibits Néel- and stripe-type fluctuations ${ }^{3}$. As such, a direct comparison between the excitations measured by INS and RIXS is not straightforward since the $\Gamma$ point is not equivalent to $M$ or $X$ in the absence of Brillouin zone folding. Nevertheless, the excitations of FeSe closely resemble those observed in $\mathrm{BaFe}_{2} \mathrm{As}_{2}{ }^{14}$, suggesting that spin fluctuations are of similar nature in these two compounds in proximity of the $\Gamma$ point and across the portion of Brillouin zone accessible to RIXS.

We observe significant differences in the RIXS spectra collected on the FeSe monolayer. At zero energy loss, we detect a strong elastic signal that likely reflects the overall diffuse scattering from the capping layer, the FeSe film, and the STO substrate. Despite this strong elastic background, we are able to identify inelastic peaks owing to the high energy resolution of the instrument
( $\sim 40 \mathrm{meV})$. In particular, we observe a broad peak located at $\sim 320 \mathrm{meV}$ at $\mathbf{q}=(0.36,0)$ r.l.u., whose energy linewidth is significantly greater than the excitations detected in the bulk case. This peak is largely asymmetric - similar to bulk FeSe - but its tail extends to energies as high as $1 \mathrm{eV}$, much higher than the bulk counterpart. Furthermore, this mode barely disperses as a function of momentum and has an energy of $\sim 320-400 \mathrm{meV}$ along the $(H, 0)$ and $(H, H)$ directions, as reported in Figs. $2 \mathrm{~d}$ and 3. Thanks to resonant photoexcitation at the $\mathrm{Fe}-L$ edge, we can identify the FeSe layer as the host of this excitation. This interpretation is further supported by the dependence of the RIXS signal on the incident photon energy across the resonance (see Supp. Inf.). The ability to make this assignment is essential to disentangle excitations originating from the film, the substrate or the interface.

The evolution of the spin excitations from FeSe bulk to monolayer is significant and cannot be compared nor ascribed to any doping effects previously observed in related materials. For example, the spin excitations of $\mathrm{BaFe}_{2} \mathrm{As}_{2}$ evolve differently depending on the doping type: in the case of hole doping (K-), the spin excitations gradually soften upon doping ${ }^{10,20}$, electron doping $(\mathrm{Co} / \mathrm{Ni}-)$ leaves the high-energy spin excitations more or less unaffected ${ }^{20-22}$ while in the isovalent doped case (P-) the spin excitations harden gradually ${ }^{15,23}$. Nonetheless, the doping- 


\section{Single-particle spectral function $A(k, E)$}
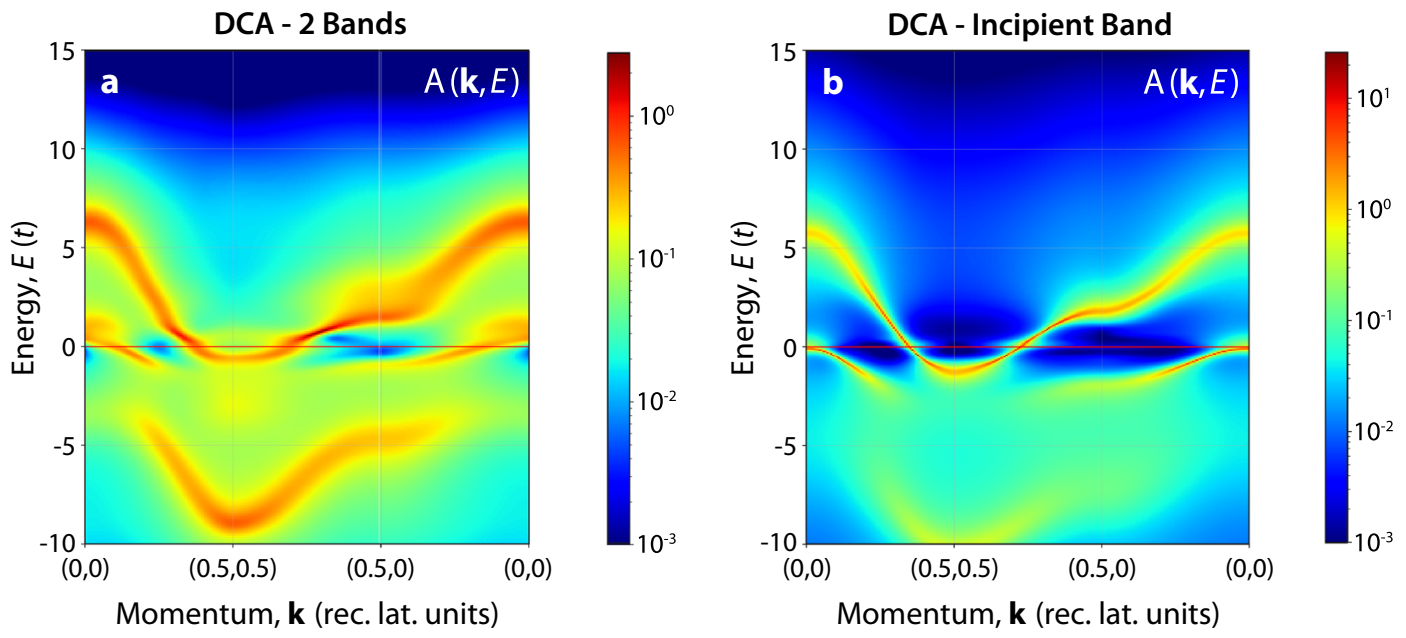

Spin susceptibility $X^{\prime \prime}(\mathbf{q}, \omega)$
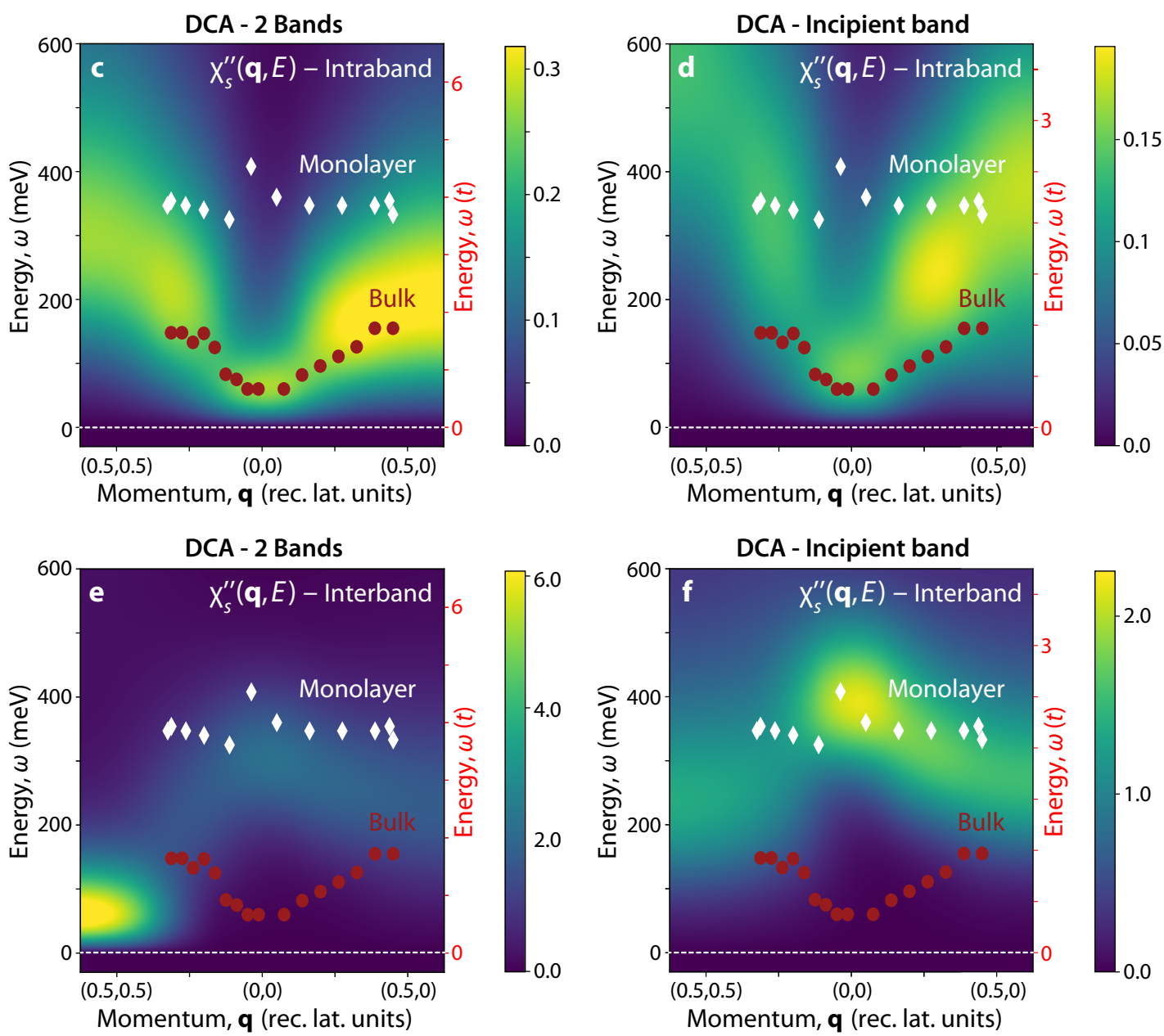

Fig. 3 Single-particle spectral function and dynamical spin susceptibility from Dynamical Cluster Approximation (DCA) calculations. a, $b$ DCA calculations and spectral function $A(\mathbf{k}, E)$ for the two-band Hubbard model (a) and the incipient band Hubbard model (b). c-f DCA calculations of the imaginary part of the spin susceptibility $\chi_{s}^{\prime \prime}(\mathbf{q}, \omega)$ for the two-band Hubbard model (c: intraband $Q_{z}=0 ;$ e interband $Q_{z}=\pi$ ) and the incipient band Hubbard model ( $\mathbf{d}$ intraband $Q_{z}=0 ; \mathbf{f}$ interband $Q_{z}=\pi$ ). Red circles (white diamonds) indicate the energy position of the peak detected by Resonant Inelastic $X$-Ray Scattering (RIXS) in bulk (monolayer) FeSe. The uncertainties associated with peak fitting are smaller than the markers. 
induced changes observed in these systems are minor compared to the effect observed here. The hardening of spin excitations in P-doped $\mathrm{BaFe}_{2} \mathrm{As}_{2}(40 \mathrm{meV})$ - so far the largest reported in the literature - is much smaller than what we observe in FeSe. Most importantly, a clear dispersion is found in these compounds at all doping levels, contrary to the flat momentum dependence in the FeSe monolayer.

Qunatum Monte Carlo calculations. The principal difference between $\mathrm{FeSe}$ and $\mathrm{FeSe} / \mathrm{STO}$ is in their band structure and Fermi surface topology. To explore the impact of these differences on the spin excitations, we calculated the single-particle spectral function $A(\mathbf{k}, E)$ and dynamical spin susceptibility $\chi_{s}^{\prime \prime}(\mathbf{q}, \omega)$ of the bilayer Hubbard model using the dynamical cluster approximation (DCA) and a nonperturbative QMC solver (see Methods). The bilayer Hubbard model is the simplest model with an electronic structure similar to the Fe-based superconductors that can be studied with QMC while maintaining a manageable sign problem. By varying the value of the nearest-neighbour interlayer hopping $t_{\perp}$, the electronic structure of the model can be tuned from a system with both hole- and electron-like bands crossing the Fermi level (Fig. 3a) to one with a single electron-like band crossing the Fermi level and an incipient hole band (Fig. 3b). The model can, therefore, capture the qualitative features of the band structure of bulk and monolayer FeSe. In Fig. 3a, we report the spectral function for the two-band model, where we observe a hole-like band crossing the Fermi level close to the $\Gamma$ point and an electron-like band intersecting the Fermi level in proximity of the $M$ point. This band structure leads to a double pocket Fermi surface as sketched in Fig. 1c. In the case of the incipient band model, shown in Fig. $3 \mathrm{~b}$, the hole band at the $\Gamma$ point is pushed to lower energies, moving below the Fermi level and removing the hole pocket at the $\Gamma$ point. The resulting Fermi surface is composed only of a circular electron pocket at the $M$ point, as sketched in Fig. 1d.

Figures $3 \mathrm{c}-\mathrm{f}$ and 4 display the calculated imaginary part of the spin susceptibility $\chi_{s}^{\prime \prime}(\mathbf{q}, \omega)$ spectra for two values of $t_{\perp}$, corresponding to bulk and FeSe/STO. In our model, two components of $\chi_{s}^{\prime \prime}(\mathbf{q}, \omega)$ are extracted with intra- $\left(q_{z}=0\right)$ and interband $\left(q_{z}=\pi\right)$ character, which can be isolated from one another by choosing the appropriate value of $q_{z}$. Figure $3 \mathrm{c}-\mathrm{f}$ report the intra- and interband channels in the middle and bottom rows, respectively. In the case of the two-band model with two ambipolar Fermi pockets, we obtain a strongly dispersing $\chi_{s}^{\prime \prime}(\mathbf{q}, \omega)$ (see Figs. 3c, e and $4 \mathrm{~b}$ ), whose main two componentsarising from intraband and interband scattering-disperse out-ofphase in momentum space. Specifically, the intraband component has a minimum at the $\Gamma$ point and increases in energy towards its maximum at $(0.5,0)$ and $(0.5,0.5)$ while the interband component displays two minima at $(0.5,0)$ and $(0.5,0.5)$ and a maximum at $(0,0)$. An analysis of the spectral intensity reveals that the interband component is four to five times larger than the intraband one. This difference is highlighted in the line cuts plot reported in Fig. 4b, where both the intra- and interband components are displayed and the former has been multiplied by a factor three for better visualization.

Upon increasing $t_{\perp}$, the hole-like band is made incipient. The interband component of the resulting $\chi_{s}^{\prime \prime}(\mathbf{q}, \omega)$ is much less dispersive and becomes gapped throughout the entire Brillouin zone, in close agreement with the experimental findings (see Figs. $3 \mathrm{f}$ and $4 \mathrm{a}$ ). The out-of-phase dispersion of the intra- and interband $\chi_{s}^{\prime \prime}(\mathbf{q}, \omega)$ is also preserved for the incipient band condition. The difference of the dispersion relation of the two susceptibilities (inter- and intra-) is larger than those observed by changing the system from a two-band model to an incipient band one. The large difference between the intra- and interband spin susceptibility is preserved even for small variations of the model parameters, as shown in the Supp. Inf. This behavior can be rationalized once one recognizes that the a channel for particlehole scattering will be closed once the hole-like band sinks below the Fermi level. Finally, we note that the width of the spectra of our $\chi_{s}^{\prime \prime}(\mathbf{q}, \omega)$ captures the broadening of the peaks in the incipient band case compared to the two-band model.

Figure $3 \mathrm{c}-\mathrm{f}$ summarize our results by comparing the calculated inter- and intra-band $\chi_{s}^{\prime \prime}(\mathbf{q}, \omega)$ as a false color image, with experimental peak positions overlaid. Here, the results are shown for both bulk (white circles) and monolayer (white diamonds) FeSe. (A more detailed description of the extraction of the experimental data points is given in the Supp. Inf.) We have assumed $t=90$ (160) $\mathrm{meV}$ for the bulk (incipient) case when converting the DCA energy scale to physical units, which produces the best agreement with the experimental data. The use of different factors for the two cases is supported by recent DMFT+LDA calculations, which indicate that bulk FeSe is more correlated than the FeSe/STO ${ }^{24}$. This conclusion is also consistent with our observation of much sharper spectral functions in the incipient band case, see Fig. 3a, b. Consequently, it is natural to adopt a larger $t$ for the monolayer case while holding the value of $U$ fixed. We note that our choice of $t=90(160) \mathrm{meV}$ corresponds to Hubbard repulsion values of $U=8 t=0.72(1.28) \mathrm{eV}$. This value is significantly renormalized down from the average value $\bar{U}=3-4 \mathrm{eV}$ obtained in first-principles calculations for the Febased superconductors ${ }^{25-27}$; however, our model value is comparable to the value $\bar{U} \approx 0.5-0.6$ obtained when the same calculations are downfolded onto a space containing only the $\mathrm{Fe}$ $3 d$ orbitals $^{25}$ by integrating out the highly polarizable $\mathrm{O} 2 p$ and As/Se $4 p$ orbitals. Our value is also consistent with those needed to reproduce the experimental value of $T_{c}$ in a recent FLEX study of the bilayer Hubbard model ${ }^{28}$. We, therefore, conclude that our energy scales are consistent with effective model treatments of the Fe-based superconductors.

Comparison of experimental data and calculations. In Fig. 4 we show a comparison of the spin susceptibility calculations with the experimental data plotted as a line cut. The experimental dispersion in bulk FeSe appears to be in better agreement with the intraband $\chi_{s}^{\prime \prime}(\mathbf{q}, \omega)$ (Fig. 3c, $\mathrm{d}$ and blue traces in Fig. $4 \mathrm{~b}$ ) rather than the interband component (Fig. 3e, $\mathrm{f}$ and orange traces in Fig. $4 b)$. The intensity of the interband $\chi_{s}^{\prime \prime}(\mathbf{q}, \omega)$ is higher than the intraband $\chi_{s}^{\prime \prime}(\mathbf{q}, \omega)$ and one might expect that the RIXS signal scales proportionally. However, matrix elements of the RIXS cross-section have not been included in the model, which makes a qualitative comparison the only viable option. Including Fe-L edge matrix elements would require a momentum-resolved full multi-orbital Fe calculation, which is currently not possible due to the severe Fermion sign problem induced by Hund's coupling. To better understand the behaviour of the proposed models and how the RIXS intensity is related to the intra- versus inter-band susceptibility, we studied a simplified ladder model using exact diagonalization. The ladder model can be viewed as a $1 \mathrm{D}$ analog to the bilayer model considered here. We compute the RIXS spectra for these ladders using the Kramers-Heisenberg formula and a relatively large cluster to obtain reasonable momentum resolution. The results (summarized in the Supp. Inf.) establish a clear connection between the RIXS intensity and the dynamical structure factor and support the use of the spin susceptibility when examining our multi-orbital model. Moreover, these simplified ladder calculations confirm that transitioning from a twoband to an incipient band model suppresses the intraband susceptibility. When taken together, these results indicate that the 
FeSe - 2 bands

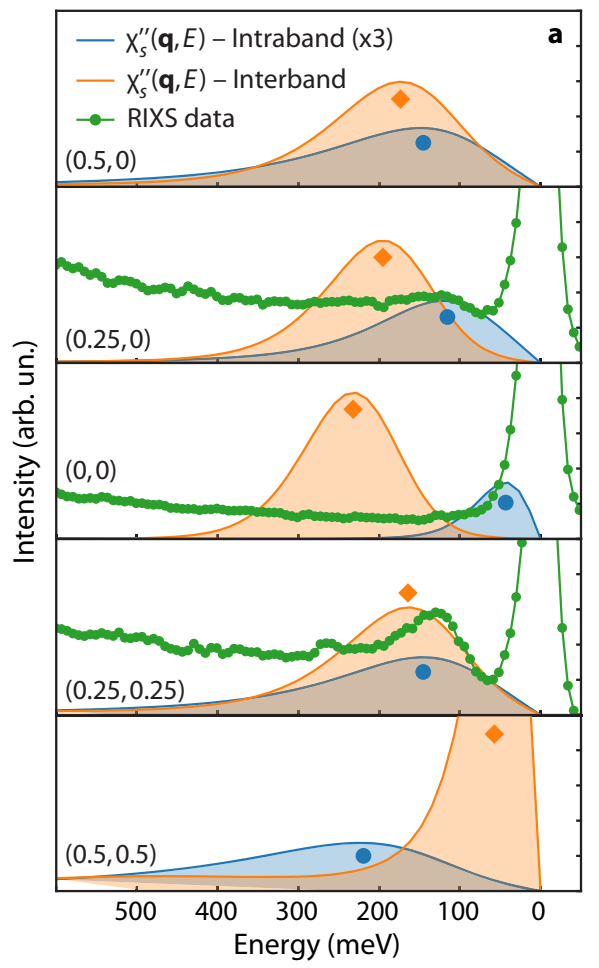

FeSe / $\mathrm{SrTiO}_{3}$ - Incipient Band

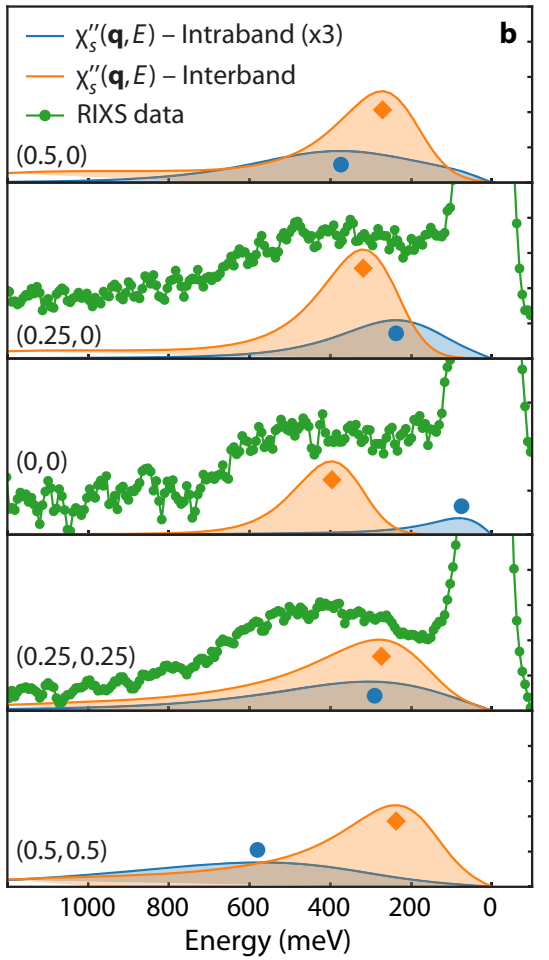

Fig. 4 Comparison of spin susceptibility and Resonant Inelastic X-Ray Scattering (RIXS) data. a Dynamical Cluster Approximation (DCA) calculations of the imaginary part of the spin susceptibility $\chi_{s}^{\prime \prime}(\mathbf{q}, \omega)$ for the incipient band Hubbard model (blue lines: intraband $k_{z}=0$; orange lines: interband $k_{z}=\pi$ ) and $\mathbf{b}$ the two-band Hubbard model (blue lines: intraband $k_{z}=0$; orange lines: interband $k_{z}=\pi$ ). Orange (blue) circles indicate the energy position of the maximum of the spin susceptibility in the interband (intraband) cases.

2D bilayer Hubbard model can be used to describe our experimental results qualitatively.

In any case, from a phenomenological perspective, the agreement of the experimental data with the intraband $\chi_{s}^{\prime \prime}(\mathbf{q}, \omega)$ from a bilayer Hubbard model is good and future calculations including orbital orientation and polarization effects could offer a more quantitative description of the RIXS cross-section. In Figs. 3d, f and $4 \mathrm{a}$, we report the calculations obtained for the incipient band model (tailored for FeSe/STO), where the agreement between theory and experiments is better for the interband $\chi_{s}^{\prime \prime}(\mathbf{q}, \omega)$ (orange lines in Fig. 4a). In this case, the interband $\chi_{s}^{\prime \prime}(\mathbf{q}, \omega)$ is flattened by the lack of the hole pocket and the hardening of the dispersion is reproduced by the theory. In Fig. 4a we also use circles to mark the two components of the spin susceptibility, which highlight the agreement between the RIXS data of Fe/STO with the interband susceptibility. These changes are a direct consequence of the fact that intraband scattering is strongly suppressed at low-energies once the hole pocket is shifted below the Fermi level. This hardening and flattening of the electronic excitations is clearly observed in the experimental data for FeSe/STO as corroborated by the white diamonds overlaid with the color plot (Fig. 3). The interband $\chi_{s}^{\prime \prime}(\mathbf{q}, \omega)$ also has the largest intensity compared to the intraband $\chi_{s}^{\prime \prime}(\mathbf{q}, \omega)$, and is, therefore, expected to dominate the RIXS signal when neglecting cross-section effects.

\section{Discussion}

Our findings have implications for the enhancement of SC in FeSe/STO. In Eliashberg- and fluctuation exchange-type models (FLEX), $\chi_{s}^{\prime \prime}(\mathbf{q}, \omega)$ enters directly into the equation to calculate $T_{\mathrm{c}}{ }^{19,29}$. The significant evolution in $\chi_{s}^{\prime \prime}(\mathbf{q}, \omega)$ revealed by our RIXS data suggests a sizable change this input of the equation, highlighting the importance of spin excitations for a complete explanation and description of SC in FeSe/STO. Moreover, any quantitative model for the spin fluctuation contribution to pairing must also account for the observed evolution of the spin dynamics. As such, the evolution of the spin dynamics from FeSe to FeSe/STO represents an essential clue to a magnetic-like pairing scenario, which was previously proposed for other Fe pnictides ${ }^{1,29-34}$. The present results do not, however, rule out additional interactions such as phonons or doping from the substrate, which can contribute to the enhancement of $T_{\mathrm{c}}^{35,36}$.

In summary, we report a combined experimental and theoretical investigation of the spin dynamics in bulk FeSe and singleunit-cell FeSe/STO, uncovering a dramatic evolution of magnetic excitations from the bulk to the monolayer limit. In bulk FeSe, we observed dispersive spin excitations that are reminiscent of other Fe-based superconductors. These modes become significantly more energetic and less dispersive in the ultrathin limit of the FeSe/STO film. Quantum Monte Carlo calculations of the bilayer Hubbard model reveal that this reconfiguration of spin dynamics is a direct consequence of the modifications to interband scattering once the hole pocket is removed from the Fermi level. These findings suggest a fundamental link between the Fermiology of FeSe superconductors and their spin dynamics up to a very high energy scale. The direct experimental insights of the present RIXS study underscore the role of spin excitations for unconventional SC in FeSe, and provide an empirical benchmark for theoretical models of SC in FeSe/STO.

\section{Methods}

\section{Sample preparation}

Monolayer FeSe on STO. Monolayer of FeSe was grown on Nb-doped (0.5 wt\%) (001)-oriented $\mathrm{SrTiO}_{3}$ substrate. The substrate was etched following the method described in ref. ${ }^{37}$. In the growth chamber, which has a base pressure of $6 \times 10^{-10}$ 
mbar, the substrate was heated to $800^{\circ} \mathrm{C}$ for 45 min with Se flux. Single-layer FeSe films were grown at $\sim 500^{\circ} \mathrm{C}$ by coevaporation of Se and $\mathrm{Fe}$ with a flux ratio of 20: 1 . After growth, the films were annealed at $550^{\circ} \mathrm{C}$ in vacuum for $2 \mathrm{~h}$. The FeSe/ STO was characterized by ARPES and the superconducting gap was determined to be $\sim 13.4 \mathrm{meV}$ or $T_{\mathrm{c}} \sim 60-65 \mathrm{~K}$. A $\sim 25 \mathrm{~nm}$ thick layer of amorphous Se was added for protection at room temperature.

FeSe bulk. Bulk FeSe single crystals were grown under a permanent gradient of temperature $\left(\sim 400-330^{\circ} \mathrm{C}\right)$ in the $\mathrm{KCl}-\mathrm{AlCl}_{3}$ flux, as reported in ref. ${ }^{3}$. The $T_{\mathrm{c}}$ of the bulk FeSe sample is $\sim 8 \mathrm{~K}$.

High energy resolution RIXS measurements on FeSe bulk and FeSe/STO. Highresolution RIXS experiments were performed at the I21-RIXS beamline at Diamond Light Source, United Kingdom. FeSe bulk was cleaved in vacuum. All samples were aligned with the surface normal (001) lying in the scattering plane. Xray absorption was measured using the total electron yield (TEY) method by recording the drain current from the samples. For RIXS measurements, $\pi$ polarized light was used. The combined energy resolution was about $40 \mathrm{meV}$ (FWHM) at the Fe $L_{3}$ edge $(\sim 710.5 \mathrm{eV})$. To enhance the RIXS throughput, a parabolic mirror has been installed in the main vacuum chamber. The RIXS spectrometer was positioned at a fixed scattering angle of 154 degrees resulting in a maximal total momentum transfer value $\mathbf{Q}$ of $\sim 0.7 \AA^{-1}$. The projection of the momentum transfer, $\mathbf{q}$, in the $a b$ plane was obtained by varying the incident angle on the sample. We use the $2 \mathrm{Fe}$ unit cell convention with $a=b=3.76 \AA$ and $c=5.4 \AA$ for the reciprocal space mapping. The momentum transfer $\mathbf{Q}$ is defined in reciprocal lattice units (r.l.u.) as $\mathbf{Q}=\mathbf{H} a^{*}+\mathbf{K} b^{*}+\mathbf{L} c^{*}$ where $a^{*}=2 \pi / a, b^{*}=2 \pi / b$, and $c^{*}=$ $2 \pi / c$. All measurements were performed at $20 \mathrm{~K}$ under a vacuum pressure of about $5 \times 10^{-10}$ mbar.

Spectra for the FeSe have been acquired in $~ 30$ min whereas spectra for the FeSe/STO required $3 \mathrm{~h}$ or more for every momentum point.

Calculations. We modeled the spin excitation spectrum of bulk and monolayer FeSe using a two-orbital Hubbard model defined on a two-dimensional square lattice with $N=L^{2}$ unit cells, where $L$ is the linear size of the system. This model includes only the intraorbital Hubbard repulsion $U$ on each orbital, and it is identical to the one used in ref. ${ }^{19}$. (Details are also provided in the Supp. Inf. for completeness). Due to the orbital symmetry of the Hamiltonian, and the restriction to only a local intra-orbital Hubbard interaction, one can regard this model as a bilayer Hubbard model with layers $\alpha=1,2^{19}$. The kinetic energy term can then be diagonalized and rewritten in terms of a bonding $k_{z}=0$ and anti-bonding $k_{z}=\pi$ basis. As such, momentum transfers with $q_{z}=0$ and $\pi$ correspond to intra- and interband excitations, respectively. Throughout, we use $t=1$ as the unit of energy, set $U=8 t$, and vary $t_{\perp}$ and the filling $n$ to control the electronic structure of the system.

We simulated the model using the dynamical cluster approximation (DCA) method ${ }^{38}$, where the bulk lattice system is mapped onto a periodic finite-size cluster embedded in a mean-field. The effective cluster problem was solved self-consistently by means of a continuous-time auxiliary field (CTAUX) quantum Monte Carlo method ${ }^{39-41}$. The real frequency dynamical correlation functions shown here were obtained from QMC data using the Maximum Entropy (MaxEnt) method ${ }^{42}$.

In our model we extracted the spectral function and dynamical susceptibilities for two different parameter sets $\frac{U}{t}=6$ and 8 . Both parameter sets produced a twoband and an incipient band model in agreement with ARPES results. The susceptibility displayed very similar trends in terms of dispersion with modifications concerning the extent of the dispersion. This corroborates that these models are robust against reasonable variations of the parameters.

We additionally performed calculations using a 1D Hubbard ladder to confirm the RIXS sensitivity to the spin susceptibility and the suppression of intraband susceptibility in the incipient band model.

\section{Data availability}

Data that support the findings of this study are available upon reasonable request from the corresponding authors.

\section{Code availability}

Codes that support the findings of this study are available upon reasonable request from the corresponding authors.

Received: 6 July 2020; Accepted: 16 April 2021; Published online: 25 May 2021

\section{References}

1. Dai, P. Antiferromagnetic order and spin dynamics in iron-based superconductors. Rev. Mod. Phys. 87, 855-896 (2015).
2. Chen, T. et al. Anisotropic spin fluctuations in detwinned FeSe. Nat. Mater. 18, 709 (2019).

3. Wang, Q. et al. Magnetic ground state of FeSe. Nat. Commun. 7, 12182 (2016)

4. Zhang, Y. et al. Superconducting gap anisotropy in monolayer FeSe thin film. Phys. Rev. Lett. 117, 117001 (2016).

5. Huang, D. \& Hoffman, J. E. Monolayer FeSe on $\mathrm{SrTiO}_{3}$. Annu. Rev. Condens Matter Phys. 8, 311-336 (2017)

6. Wang, Q.-Y. et al. Interface-induced high-temperature superconductivity in single unit-cell fese films on $\mathrm{SrTiO}_{3}$. Chin. Phys. Lett. 29, 037402 (2012).

7. Peng, R. et al. Tuning the band structure and superconductivity in single-layer FeSe by interface engineering. Nat. Commun. 5, 1-7 (2014).

8. Ding, $\mathrm{H}$. et al. High-temperature superconductivity in single-unit-cell FeSe films on anatase $\mathrm{TiO}_{2}$ (001). Phys. Rev. Lett. 117, 067001 (2016).

9. Lee, D.-H. Routes to high-temperature superconductivity: a lesson from FeSe/ $\mathrm{SrTiO}_{3}$. Annu. Rev. Condens. Matter Phys. 9, 261-282 (2018).

10. Zhou, K.-J. et al. Persistent high-energy spin excitations in iron-pnictide superconductors. Nat. Commun. 4, 1470 (2013).

11. Pelliciari, J. et al. Presence of magnetic excitations in SmFeAsO. Appl. Phys. Lett. 109, 122601 (2016).

12. Pelliciari, J. et al. Intralayer doping effects on the high-energy magnetic correlations in NaFeAs. Phys. Rev. B 93, 134515 (2016).

13. Pelliciari, J. et al. Local and collective magnetism of EuFe $\mathrm{As}_{2}$. Phys. Rev. B 95, 115152 (2017).

14. Rahn, M. C. et al. Paramagnon dispersion in $\beta$-FeSe observed by Fe L-edge resonant inelastic x-ray scattering. Phys. Rev. B 99, 014505 (2019).

15. Pelliciari, J. et al. Reciprocity between local moments and collective magnetic excitations in the phase diagram of $\mathrm{BaFe}_{2}\left(\mathrm{As}_{1-x} \mathrm{P}_{x}\right)_{2}$. Commun. Phys. 2, 139 (2019).

16. Garcia, F. A. et al. Anisotropic magnetic excitations and incipient N'eel order in $\mathrm{Ba}\left(\mathrm{Fe}_{1-x} \mathrm{Mn}_{x}\right)_{2} \mathrm{As}_{2}$. Phys. Rev. B 99, 115118 (2019).

17. Ament, L. J. P., van Veenendaal, M., Devereaux, T. P., Hill, J. P. \& van den Brink, J. Resonant inelastic x-ray scattering studies of elementary excitations. Rev. Mod. Phys. 83, 705-767 (2011).

18. Dean, M. P. M. Insights into the high temperature superconducting cuprates from resonant inelastic X-ray scattering. J. Magn. Magn. Mater. 376, 3-13 (2015).

19. Maier, T. A. \& Scalapino, D. J. Pair structure and the pairing interaction in a bilayer Hubbard model for unconventional superconductivity. Phys. Rev. B 84, 180513 (2011).

20. Wang, M. et al. Doping dependence of spin excitations and its correlations with high-temperature superconductivity in iron pnictides. Nat. Commun. 4, 2874 (2013).

21. Luo, H. et al. Electron doping evolution of the anisotropic spin excitations in $\mathrm{BaFe}_{2-x} \mathrm{Ni}_{x} \mathrm{As}_{2}$. Phys. Rev. B 86, 024508 (2012).

22. Luo, $\mathrm{H}$. et al. Electron doping evolution of the magnetic excitations in $\mathrm{BaFe}_{2-x} \mathrm{Ni}_{x} \mathrm{As}_{2}$. Phys. Rev. B 88, 144516 (2013).

23. $\mathrm{Hu}, \mathrm{D}$. et al. Spin excitations in optimally $\mathrm{P}$-doped $\mathrm{BaFe}_{2}\left(\mathrm{As}_{0.7} \mathrm{P}_{0.3}\right)_{2}$ superconductor. Phys. Rev. B 94, 094504 (2016).

24. Mandal, S., Zhang, P., Ismail-Beigi, S. \& Haule, K. How correlated is the FeSe/ $\mathrm{SrTiO}_{3}$ system? Phys. Rev. Lett. 119, 067004 (2017).

25. Anisimov, V. I. et al. Coulomb repulsion and correlation strength in $\mathrm{LaFeAsO}$ from density functional and dynamical mean-field theories. J. Phys.: Condens. Matter 21, 075602 (2009).

26. van Roekeghem, A., Vaugier, L., Jiang, H. \& Biermann, S. Hubbard interactions in iron-based pnictides and chalcogenides: slater parametrization, screening channels, and frequency dependence. Phys. Rev. B 94, 125147 (2016).

27. Watson, M. D. et al. Formation of Hubbard-like bands as a fingerprint of strong electron-electron interactions in FeSe. Phys. Rev. B 95, 081106 (2017).

28. Rademaker, L., Alvarez-Suchini, G., Nakatsukasa, K., Wang, Y. \& Johnston, S. Enhanced superconductivity in $\mathrm{FeSe} / \mathrm{SrTiO}_{3}$ from the combination of forward scattering phonons and spin fluctuations. Phys. Rev. B 103, 144504 (2021).

29. Linscheid, A., Maiti, S., Wang, Y., Johnston, S. \& Hirschfeld, P. High $\mathrm{T}_{c}$ via spin fluctuations from incipient bands: application to monolayers and intercalates of FeSe. Phys. Rev. Lett. 117, 077003 (2016).

30. Chubukov, A. Pairing mechanism in Fe-based superconductors. Annu. Rev. Condens. Matter Phys. 3, 57-92 (2012).

31. Chubukov, A. V. Itinerant electron scenario for Fe-based superconductors (2015). https://arxiv.org/abs/1507.03856.

32. Chubukov, A. \& Hirschfeld, P. J. Iron-based superconductors, seven years later. Phys. Today 68, 46-52 (2015).

33. Mishra, V., Scalapino, D. J. \& Maier, T. A. $s_{ \pm}$pairing near a lifshitz transition. Sci. Rep. 6, 32078 (2016).

34. Shigekawa, K. et al. Dichotomy of superconductivity between monolayer FeS and FeSe. Proc. Natl Acad. Sci. USA 116, 24470-24474 (2019).

35. Lee, J. J. et al. Interfacial mode coupling as the origin of the enhancement of $\mathrm{T}_{c}$ in FeSe films on $\mathrm{SrTiO}_{3}$. Nature 515, 245-248 (2014). 
36. Zhang, C. et al. Ubiquitous strong electron-phonon coupling at the interface of $\mathrm{FeSe} / \mathrm{SrTiO}_{3}$. Nat. Commun. 8, 14468 (2017).

37. Tan, S. et al. Interface-induced superconductivity and strain-dependent spin density waves in $\mathrm{FeSe} / \mathrm{SrTiO}_{3}$ thin films. Nat. Mater. 12, 634-640 (2013).

38. Maier, T., Jarrell, M., Pruschke, T. \& Hettler, M. H. Quantum cluster theories. Rev. Mod. Phys. 77, 1027-1080 (2005).

39. Hähner, U. R. et al. DCA++: A software framework to solve correlated electron problems with modern quantum cluster methods. Comput. Phys. Commun. 246, 106709 (2020).

40. Gull, E., Werner, P., Parcollet, O. \& Troyer, M. Continuous-time auxiliary-field Monte Carlo for quantum impurity models. Europhys. Lett. 82, 57003 (2008).

41. Gull, E. et al. Submatrix updates for the continuous-time auxiliary-field algorithm. Phys. Rev. B 83, 075122 (2011).

42. Gubernatis, J. E., Jarrell, M., Silver, R. N. \& Sivia, D. S. Quantum Monte Carlo simulations and maximum entropy: dynamics from imaginary-time data. Phys. Rev. B 44, 6011-6029 (1991).

\section{Acknowledgements}

We acknowledge John Tranquada, Rafael Fernandes, Connor Occhialini, and Andrey Chubukov for enlightening discussions. We also thank Nick Brookes, Kurt Kummer, and Davide Betto for initial tests on FeSe/STO. This work was supported by the Air Force Office of Scientific Research Young Investigator Program under grant FA9550-19-10063 (J.P. and R.C.). We thank Diamond Light Source for the allocation of beamtime to proposal SP18883. J.P. acknowledges financial support by the Swiss National Science Foundation Early Postdoc Mobility Fellowship Project No. P2FRP2 171824 and P400P2_180744. S.K., T.A.M., and S.J. are supported by the Scientific Discovery through Advanced Computing (SciDAC) program funded by U.S. Department of Energy, Office of Science, Advanced Scientific Computing Research and Basic Energy Sciences, Division of Materials Sciences and Engineering. S.J. acknowledges additional support from the Office of Naval Research under Grant No. N00014-18-1-2675. An award of computer time was provided by the INCITE program. This research also used resources of the Oak Ridge Leadership Computing Facility, which is a DOE Office of Science User Facility supported under Contract DE-AC05-00OR22725. M.R. and G.G. were supported by the ERC-P-ReXS project (2016-0790) of the Fondazione CARIPLO and Regione Lombardia, in Italy. R.A. is supported by the Swedish Research Council (VR) under the Project 201700382. R.C. acknowledges support from the Alfred P. Sloan Foundation. X. C, R.P. and D.L.F acknowledge the support from National Natural Science Foundation of China (Nos. 11790310 and 11922403). R.C. and G.G. acknowledge support from the MITPOLIMI Program (Progetto Rocca) of the MIT International Science and Technology Initiatives (MISTI) and Politecnico di Milano.

\section{Author contributions}

J.P., R.A., A.N., M.R., J.L., M.G.F., G.G., A.C.W., K.Z. performed the RIXS experiments. Q.S., T.Y., X.C., R.P., D.F. prepared the FeSe/STO thin films. Q.W. and J.Z. grew FeSe single crystals. S.K., S.J., and T.A.M. performed the theory calculations. J.P., S. K., S.J., T.A.M., and R.C. wrote the manuscript with input from all the authors. R.C. supervised the project.

\section{Competing interests}

The authors declare no competing interests.

\section{Additional information}

Supplementary information The online version contains supplementary material available at https://doi.org/10.1038/s41467-021-23317-3.

Correspondence and requests for materials should be addressed to J.P. or R.C.

Peer review information Nature Communications thanks the anonymous reviewer(s) for their contribution to the peer review of this work.

Reprints and permission information is available at http://www.nature.com/reprints

Publisher's note Springer Nature remains neutral with regard to jurisdictional claims in published maps and institutional affiliations.

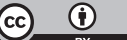

Open Access This article is licensed under a Creative Commons Attribution 4.0 International License, which permits use, sharing, adaptation, distribution and reproduction in any medium or format, as long as you give appropriate credit to the original author(s) and the source, provide a link to the Creative Commons license, and indicate if changes were made. The images or other third party material in this article are included in the article's Creative Commons license, unless indicated otherwise in a credit line to the material. If material is not included in the article's Creative Commons license and your intended use is not permitted by statutory regulation or exceeds the permitted use, you will need to obtain permission directly from the copyright holder. To view a copy of this license, visit http://creativecommons.org/ licenses/by/4.0/.

(C) The Author(s) 2021 